\title{
Statistical Electron Excitation in a DQD Induced by Two Independent Quantum Point Contacts
}

\author{
U. Gasser, S. Gustavsson, B. Küng, K. Ensslin, and T. Ihn \\ Solid State Physics Laboratory, ETH Zurich, CH-8093 Zurich, Switzerland \\ D.C. Driscoll and A.C. Gossard \\ Materials Department, University of California, Santa Barbara, California 93106, USA
}

(Dated: November 3, 2018)

\begin{abstract}
We investigate experimentally the influence of current flow through two independent quantum point contacts to a nearby double quantum dot realized in a GaAs-AlGaAs heterostructure. The observed current through the double quantum dot can be explained in terms of coupling to a bosonic bath. The temperature of the bath depends on the power generated by the current flow through the quantum point contact. We identify the dominant absorption and emission mechanisms in a double quantum dot as an interaction with acoustic phonons. The experiment excludes coupling of a double quantum dot to shot-noise generated by quantum point contact as the dominant mechanism.
\end{abstract}

\section{INTRODUCTION}

Electronic transport through semiconductor double quantum dots (DQDs) has been intensively explored for nearly two decades, $1,2,3$ The interplay between a double quantum dot and its environment was investigated in detail in previous works $4,5,6,7,8,9,10$ using microwave spectroscopy. Irradiating double quantum dots with microwaves results in photon assisted tunneling (PAT) 11 The integration of a quantum point contact (QPC) in the vicinity of a single quantum dot allowed charge detection, ${ }^{12}$ which was later implemented in double quantum dot systems. 13,14

The novel application of a quantum point contact as a source of energy to drive inter-dot electronic transitions in a double quantum dot was recently realized,15,16,17 These experiments were explained in terms of acoustic phonon based energy transfer between the QPC and the DQD circuits. The combination of a capacitatively coupled DQD-QPC system with time resolved charge detection resulted in a frequency-selective detector for microwave radiation. It allows to detect single photons emitted by the QPC and absorbed by the DQD. 18

Understanding the back-action of a charge sensor on a DQD is important for future possible applications in quantum information processing. 19 The possible dominant mechanisms that lead to QPC-induced inter-dot electronic transitions include electron scattering with photons $^{18}$ and acoustic phonons ${ }^{20}$ or shot-noise ${ }^{21,22}$ depending on the parameter regime investigated.

In this paper we study back-action of the current flow through the QPC detector on a serial double dot. The double dot is tuned to an asymmetric regime, where one dot is strongly coupled to the source lead, whereas the second dot is more weakly coupled to the drain lead. Two independent QPCs can be simultaneously used for driving the transitions in the DQD. We observe a nonadditive effect of both QPCs accompanied by the saturation of the current across the double quantum dot for large QPC currents. We explain the measured data in the framework of interaction of electrons with acoustic phonons. We relate the power emitted by the QPC to the temperature of the phononic bath. The experiment excludes the possibility of shot-noise being the source of inter-dot transitions.

This paper is organized as follows. In Sec. II we describe the fabrication of the sample, its electrostatic characterization and functionality. In Sec. III we present a detailed description of the working regime of a DQD and QPCs, followed by the results of our measurements of current through a DQD using one and two QPCs. We discuss the possible interaction mechanisms in Sec. IV. In Sec.V $\mathrm{V}$ we introduce a model based on electron-phonon interaction and in Sec.VI we interpret the measured data. Section VII contains the conclusions.

\section{SAMPLE AND CHARACTERIZATION}

The sample shown in Fig. 11(a) is based on a $\mathrm{GaAs} / \mathrm{Al}_{0.3} \mathrm{Ga}_{0.7} \mathrm{As}$ heterostructure with a twodimensional electron gas (2DEG) $34 \mathrm{~nm}$ below the surface. It was fabricated by double layer local oxidation with a scanning force microscope (SFM) 23 The 2DEG is depleted below the oxide lines written on the GaAs surface $^{24}$ [white lines in Fig. 11(a)]. A 4 nm titanium film was evaporated and patterned by local oxidation to create mutually isolated top gates [indicated by the dashed lines in Fig. 1(a)].

The confinement potential produced by the top gates and the oxide lines is shown in the contour plot in Fig. 1(b). It was calculated assuming a pinned surface model $^{25}$ using the lithographic sizes of the gates measured after the sample was fabricated. It shows an approximately circular symmetry for the dots, with the left quantum dot being slightly larger than the right one. The color scale is in arbitrary units 26

The structure presented in Fig. 1(a) consists of three electronic circuits. The first one is formed by two quantum dots connected in series [marked by the grey (red 
(a)

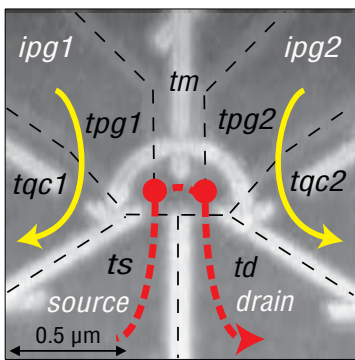

(b)

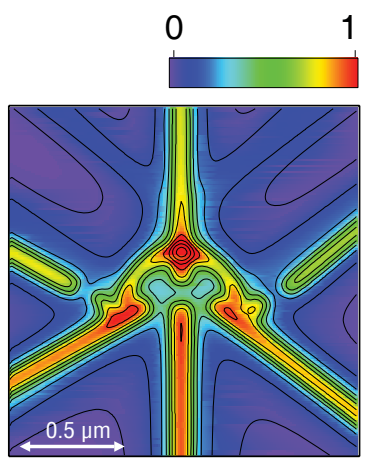

(c)

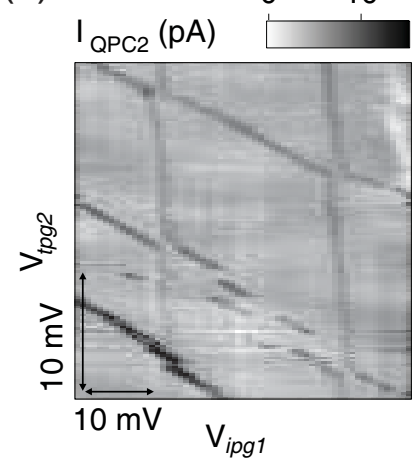

(d)

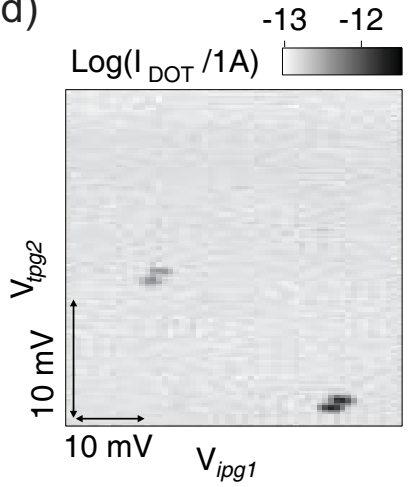

FIG. 1: (Color online) (a) SFM image of the sample. In-plane gates are defined by the thick white lines, titanium oxide lines are indicated by dashed lines. Top gates are labeled with black letters. The QDs are indicated with grey (red online) circles. The white (yellow online) arrows indicate the positive direction of the current in the QPCs. (b) Calculated electrostatic potential in the 2DEG generated by the oxide lines and the top gates. (c) Modulated current component proportional to the transconductance measured through QPC2 for $\mathrm{V}_{\mathrm{QPC} 2}=0.5 \mathrm{mV}$. An AC voltage of $1.5 \mathrm{mV}$ with a frequency of $34 \mathrm{~Hz}$ was applied to the in-plane gate ipg1 and detected in the QPC2 circuit using lock-in techniques. The honeycomb pattern and four pairs of triple points are visible. (d) Simultaneously measured double dot DC current. The source-drain voltage was $\mathrm{V}_{S D}=60 \mu \mathrm{V}$.

online) circles] and connected to source and drain. A negative DQD current corresponds to electrons moving from source to drain. Each of the other two circuits contains a quantum point contact [white (yellow online) solid arrows]. A negative QPC current means electrons traveling through the QPC in the direction of the arrows.

To sum up, our structure consists of two barriers defining quantum point contacts, two quantum dots, two barriers determining the coupling of the quantum dots to the source and drain and the barrier that determines the coupling between the quantum dots. In total, this gives seven degrees of freedom and there are seven independent top-gates used to tune these barriers and the quantum dots. The top gates (tpg1 and tpg2) are used to tune the DQD into a suitable regime. The top gates $t q c 1$ and tqc2 can tune the transmission of QPC1 and QPC2, re- spectively. The middle top gate $t m$ controls the coupling between the two dots allowing to change smoothly from the single dot regime (large dot spread over the area covered by the two red circles) to a weakly-coupled double dot. The gates $t s$ and $t d$ are used to tune the coupling of the DQD to source and drain.

The potential on both sides of QPC1 (QPC2) can be lifted with respect to the measurement ground, creating a mutual gating effect between DQD and QPC1 (QPC2). These in-plane gates (ipg1 for QPC1 and ipg2 for QPC2) control the number of electrons on the DQD.

Due to the presence of the metallic top gates, the electrostatic interaction between electrons in the quantum dots and the QPCs is weakened by screening compared to semiconductor-only quantum circuits ${ }^{27}$ The large distance between the QPC and the double dot (lithographic distance $450 \mathrm{~nm}$ ) further reduces the sensitivity of the QPC for detecting electrons passing through the DQD.

Figures 1(c) and (d) demonstrate the operation of QPC2 as a charge detector $\stackrel{12}{=}$ For both QPCs, the onedimensional subband spacing is larger than $3.5 \mathrm{mV}$ as estimated from finite bias measurements. In order to use QPC2 (QPC1) as a charge read-out, its conductance was tuned to $e^{2} / h$. A constant voltage of $0.5 \mathrm{mV}$ was applied between the source and drain leads of the QPC, and the current was measured. An AC voltage of $1.5 \mathrm{mV}$ applied at $34 \mathrm{~Hz}$ to the opposite in-plane gate ipg1 (ipg2) modulated the current through QPC2 (QPC1). This modulated signal which is proportional to the transconductance was detected with lock-in techniques. The measurements were performed in a dilution refrigerator at a base temperature of $70 \mathrm{mK}$.

The resulting stability diagram of the DQD detected with QPC2 is shown in Fig. 1(c). The boundaries between regions of different ground state charge configurations of the DQD are clearly visible. In this measurement, tpg2 is used to change the number of electrons in the right dot and ipg1 to change the number of electrons in the left dot. A few charge rearrangements in the lower half of the honeycomb induced by the metallic top gate tpg1 are present. In general, we find that the top gate sweeps lead to significantly more charge rearrangements than sweeps of the in-plane gates. Change of the ipg2 potential combined with simultaneous charge detection would result in strong detuning of the charge sensor. This would shift the operating point far away from the sensitive regime. It can be avoided by using tpg2 because it has a weaker lever arm on the QPC. The thick line in the bottom-left corner of the plot corresponds to a resonance in $\mathrm{QPC} 2$.

In Fig. 1(d) the corresponding DC current through the DQD is plotted. It was measured simultaneously with the QPC signal presented in the previous paragraph. The source-drain voltage applied to the DQD is $60 \mu \mathrm{V}$. Only two pairs of triple points are visible. Similar sets of data can be obtained using QPC1 as the detector. 

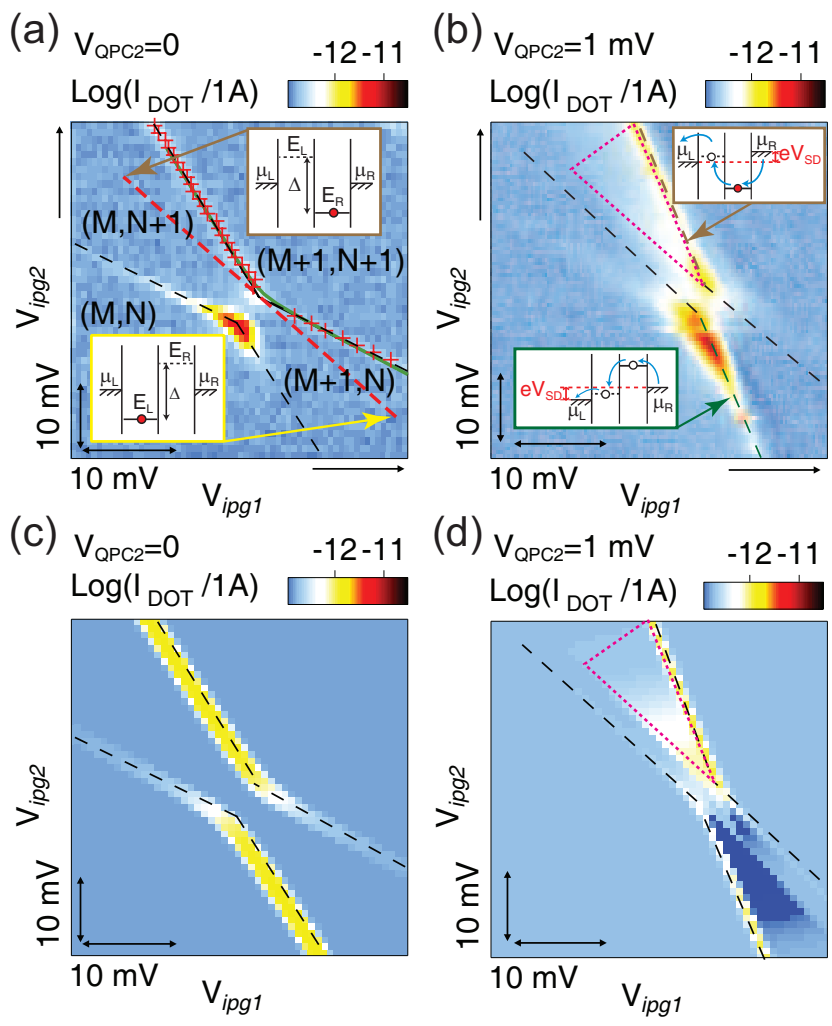

FIG. 2: (Color online) (a) Double dot current as a function of the gate voltages $V_{i p g 1}$ and $V_{i p g 2}$. It represents the charge stability diagram of the DQD. The dashed lines outline the edges of the honeycomb cells. ${ }^{2} \mathrm{M}$ and $\mathrm{N}$ refer to the number of electrons in the left and the right dot, respectively. The red line denotes the detuning axis, with zero detuning occurring at the triple point. The red crosses correspond to the positions of the current maxima. The green line depicts the calculated charge configuration diagram with the tunneling coupling $t=$ $50 \mu \mathrm{eV}$. It was obtained assuming the constant interaction model with a finite tunnel coupling. $\frac{2}{-1}$ The data was taken with $V_{\mathrm{SD}}=100 \mu \mathrm{V}$ applied symmetrically across the DQD, lifting the chemical potential in the source lead $\mu_{\mathrm{L}}$ up and lowering the energy of the drain lead $\mu_{\mathrm{R}}$. The insets schematically show the level alignment at points indicated by the arrows. (b) The same region as in (a) but with a $1 \mathrm{mV}$ DC bias voltage applied across QPC1 and $60 \mu \mathrm{V}$ applied symmetrically across the DQD. The triangle indicates the region with the induced DQD current. The insets show schematic diagrams of the electrochemical potentials in the left and the right quantum dot along the selected honeycomb boundaries. (c) Calculated current through a double dot as a function of gate voltages. The calculation refers to the measurement presented in panel (a), where no bias voltage was applied to the QPC. The details of the calculation are discussed in the text. (d) Calculated stability diagram corresponding to the situations in (b), where a voltage of $1 \mathrm{mV}$ was applied across the QPC2. The dark blue color in (d) was used to mark the region with a negative current. The comparison between (b) and (d) is presented in the text.

\section{EXPERIMENTAL DATA}

In the following, we concentrate on a single pair of triple points where the DQD showed moderate coupling. Figure 2(a) shows the DC DQD current $\left(\mathrm{I}_{\mathrm{DOT}}\right)$ for $100 \mu \mathrm{eV}$ source-drain bias applied across the DQD. The inter-dot mutual capacitance $C_{m}$ estimated from finite bias measurements and from the stability diagram assuming the constant interaction model ${ }^{2}$ is $8.8 \mathrm{aF}$, whereas the total capacitance of the left dot is $C_{1}=86 \mathrm{aF}$ and for the right dot, $C_{2}=76 \mathrm{aF}$. Each dot contains approximately 15 electrons and the charging energies are about $2 \mathrm{meV}$.

The thin dashed lines in Fig. 2(a) indicate the boundaries of the honeycomb pattern and the numbers in brackets $(\mathrm{M}, \mathrm{N})$ denote the charge population of the left and the right quantum dot, respectively. Here, the left dot is strongly coupled to the source lead, whereas the right dot is weakly coupled to the drain reservoir. During the measurement, both QPCs were kept at zero bias.

The detuning marked by the dashed grey (red online) line in Fig. 2(a) is obtained from the capacitance mode ${ }^{2}$ and expressed by the equation $\delta=E_{\mathrm{L}}-E_{\mathrm{R}}$ such that the total energy of the DQD, $E_{\text {tot }}=E_{L}+E_{R}$ remains constant. The energies $E_{L}$ and $E_{R}$ are the single-particle energies in the left and the right quantum dot, respectively. Converting the energies to gate voltages gives: $\delta=\left(\alpha_{i p g 1, R}-\alpha_{i p g 1, L}\right) \Delta V_{i p g 1}+\left(\alpha_{i p g 2, R}-\alpha_{i p g 2, L}\right) \Delta V_{i p g 2 .}$. The lever arms $\alpha_{i p g 1, j}$ and $\alpha_{i p g 2, j}$ are the lever arms of the in-plane gates ipg2 and ipg2 on the left $(j=L)$ or the right $(j=R)$ dot, respectively. The voltages $V_{i p g 1}$ and $V_{i p g 2}$ are the voltages applied to the gates ipg1 and ipg2. The lever arms are extracted from measurements at finite bias and from the charge stability diagram of the DQD. The obtained values are: $\alpha_{i p g 1, R}=0.048, \alpha_{i p g 1, L}=0.021$, $\alpha_{i p g 2, R}=0.03$ and $\alpha_{i p g 2, L}=0.04$. We take zero detuning to occur at the triple point. According to the definition above, detuning is positive (negative) in the upper-left (lower right) part of Fig. 2(a). Two representative energy diagrams are shown in the insets.

In Fig. 2(b) the DQD current was measured in the same parameter range at a QPC2 bias voltage of $1 \mathrm{mV}$. The bias voltage across DQD was set to $60 \mu \mathrm{eV}$, i.e., smaller than the bias voltage applied in Fig.2(a). Despite that, the current is strongly enhanced along the boundaries $(\mathrm{M}, \mathrm{N}) \rightarrow(\mathrm{M}+1, \mathrm{~N})$ and $(\mathrm{M}, \mathrm{N}+1) \rightarrow(\mathrm{M}+1, \mathrm{~N}+1)$, corresponding to adding an electron to the left dot. The enhancement of the current along the honeycomb boundaries is induced by driving a current through QPC2. Another visible feature induced by biasing QPC2 is the finite DQD current in the triangle-shaped area indicated in Fig. 2(b) that is normally forbidden by Coulomb blockade.

The following measurements were carried out with the QPCs tuned to their first conductance plateau. The overall experimental results do not depend on this operation point of the QPC.

To investigate the influence of the QPC currents on the DQD in the triangular region, we tuned the levels in the 

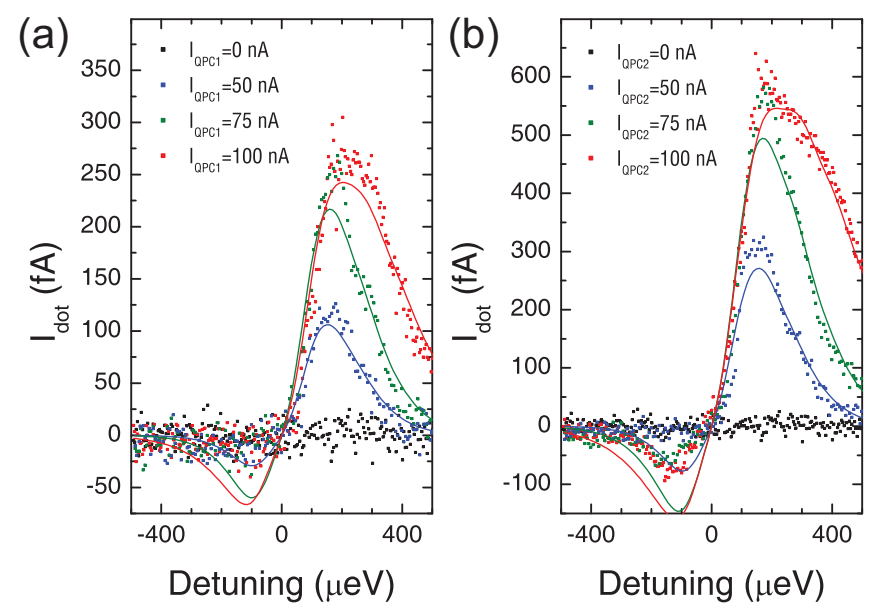

FIG. 3: Current through the DQD along the detuning line: (a) for different QPC1 currents and (b) different QPC2 currents. Fits are plotted with solid lines (see text).

dot along the detuning line depicted as the solid red line in Fig.2(a). Fig. 3] shows the dot current versus detuning. The black data points in Fig. 3(a) were taken with zero bias applied to the DQD as well as to QPC1 and QPC2. No measurable current above the noise level is detected. When a DC current of $50 \mathrm{nA}$ is driven through QPC1, an asymmetric peak with a maximum of about $125 \mathrm{fA}$ along the detuning line is observed (blue points in Fig. 3(a)). This effect is strongly enhanced if the current through QPC1 is further increased to $75 \mathrm{nA}$ (green points) and $100 \mathrm{nA}$ (red points). All traces cross zero at the triple point (zero detuning).

A similar, but significantly more pronounced effect is observed if QPC2 is driven, as shown in Fig. 3(b). Moreover, for negative detuning a small negative DQD current is observed. QPC2 is more sensitive as a charge readout and it has a stronger effect on the DQD. Therefore, we conclude that QPC2 is more strongly coupled to the DQD than QPC1.

We have chosen one point on the detuning line corresponding to $\delta=200 \mu \mathrm{eV}$ and swept the QPC1 and QPC2 currents. The results of this measurement are shown in Fig. 廿(a). The black (red) filled squares correspond to positive currents through QPC1 (QPC2) swept from 0 to $200 \mathrm{nA}$. The empty black (red) circles are the traces recorded while the QPC1 (QPC2) current was swept from 0 to $-200 \mathrm{nA}$. The QPC induced DQD current is a little larger in the case when the QPCs are driven with positive current. This polarity dependence is significant and we can exclude that it is due to a gating effect. As mentioned before, QPC2 is more strongly coupled to the DQD than QPC1. When the QPC current is swept in a positive direction (filled symbols in Fig. (4) the DQD current starts to level off (the inflection points for are lying between 100 and $150 \mathrm{nA}$ in the QPC current axis), whereas for negative QPC current directions this effect is not clearly visible.
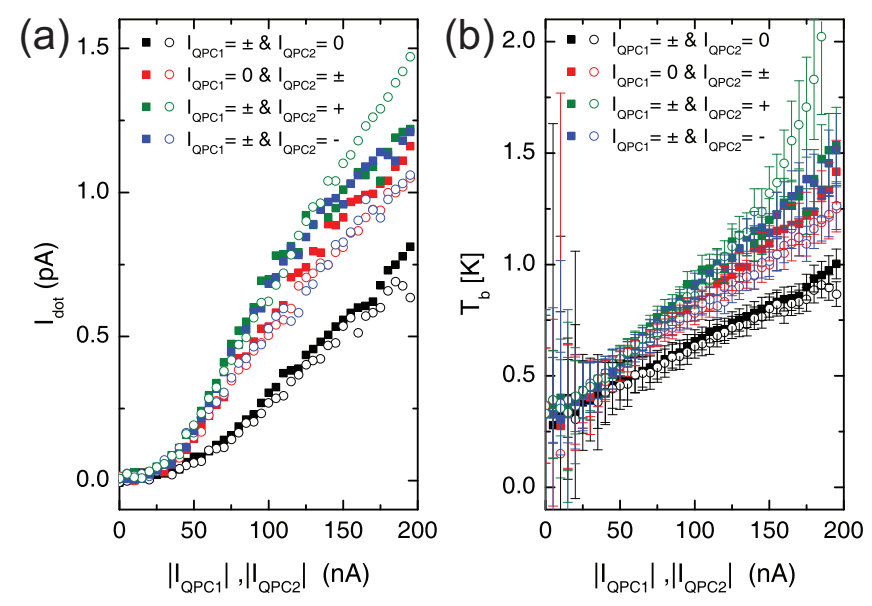

FIG. 4: (a) Current through the DQD at fixed detuning of $200 \mu \mathrm{eV}$ as a function of QPC1 and QPC2 current. The open and closed symbols correspond to the "+" and "-" sign in the corresponding expression, respectively. (b) Temperature of the bosonic bath as a function of the QPC1 and QPC2 current calculated from (a) (see text).

Another unexpected feature is observed on the green traces. The filled (empty) green squares correspond to the QPC1 current being swept from 0 to $200(-200) \mathrm{nA}$ while the QPC2 current is simultaneously swept from 0 to $200 \mathrm{nA}$. In a simple picture, we would expect that the effects of QPC1 and QPC2 are independent and they add up, but the measurement contradicts this expectation. Due to the action of both QPCs the DQD current is slightly larger than in the case when only QPC2 is used. In addition, there is an unexpected polarity dependence with a maximum DQD current for QPC1 being swept in negative and QPC2 in positive direction. The remaining

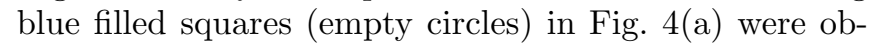
tained by driving a positive (negative) current through $\mathrm{QPC} 1$ and a negative current through QPC2.
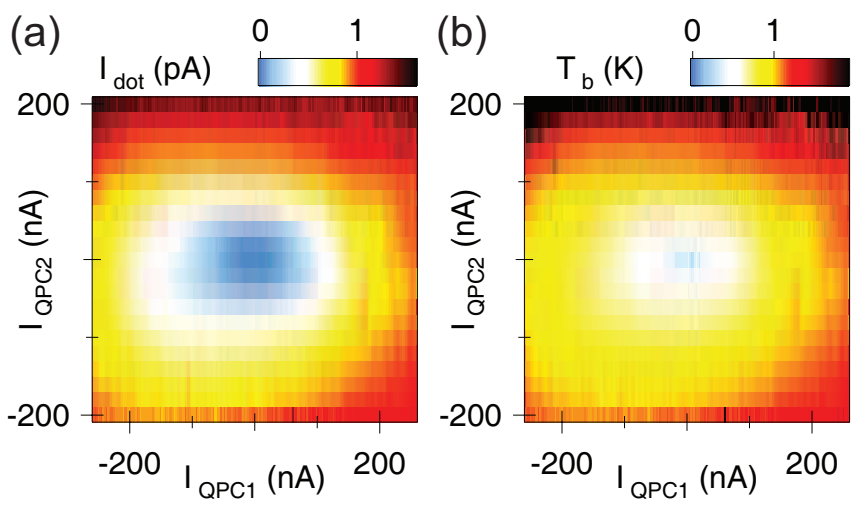

FIG. 5: (Color online) (a) The DQD current as a function of the QPC1 and QPC2 currents. The data was taken at a fixed detuning $\delta=200 \mu \mathrm{eV}$. (b) Calculated temperature of the bosonic bath $T_{\mathrm{b}}$.

The polarity effect in the DQD current is also present 
in Fig. 5(a), where the dot current was plotted versus QPC1 and QPC2 currents at fixed detuning $\delta=200 \mu \mathrm{eV}$. In this measurement, the lack of additivity of the effects induced by the QPCs is even more visible.

\section{DISCUSSION OF POSSIBLE MECHANISMS}

A mechanism which can induce the current flow through the double dot along the detuning line is presented in Fig. 6(a). The driving current through QPC1 or/and QPC2 is thought to lead to an emission of energy, which can be absorbed by the electron in the right dot. If the provided energy matches the energy difference $\Delta$, the electron can be excited from the right to the left dot. If the electron leaves the DQD through the left lead and the next electron tunnels into the right dot through the right lead, then the cycle closes and there is a measurable current flowing through the double dot.

An additional enhancement of the DQD current along the honeycomb boundaries as observed in Fig. 2(b), induced by driving a current through a QPC, can be explained in a similar way. In the situation shown in the upper inset the electron trapped in the right quantum dot can absorb energy emitted by the QPC2, tunnel into the left dot and leave the DQD system via the left lead. The cycle closes when the next electron tunnels into the right dot from the right lead. This QPC2 induced process gives an additional contribution to the DQD current. This effect is more pronounced in the vicinity of the triple point where energy difference between the levels $E_{L}$ and $E_{R}$ in the left and the right dots are small. The lower inset of Fig. 2(b) shows the analogous diagram for the situation when the level in the right quantum dot lies above the Fermi energy of the leads. Again, the QPC2 induced process causes the electrons to move from the right into the left contact.

The possible mechanisms of the pumping effect are coupling to acoustic or optical phonons, plasmons, photons, shot-noise or thermopower effect. Scattering with optical phonons is strongly suppressed as long as the relevant energy scales are smaller than the optical phonon energy 28 Coupling to plasmons can be ruled out as well. 20 We can also exclude the shot-noise as a source of the energy, because during the experiment both QPCs were tuned to their first plateau. Measurements performed at $0.5 G_{0}$ and $1.5 G_{0}\left(G_{0}=2 e^{2} / h\right)$ showed a qualitatively and quantitatively similar behavior. This is in contrast to previously measured data ${ }^{15,21}$ where no DQD current was observed in the plateau regions. Coupling to acoustic phonons is the most likely mechanism of inducing the current in the DQD. Further below in this paper, we discuss the data in the light of phonon coupling and a related thermopower effect.

The questions arising from the data presented above are the following: is the strong difference of the peak heights on the positive and negative side of the detuning (Fig. 3) due to the asymmetry in the DQD coupling to

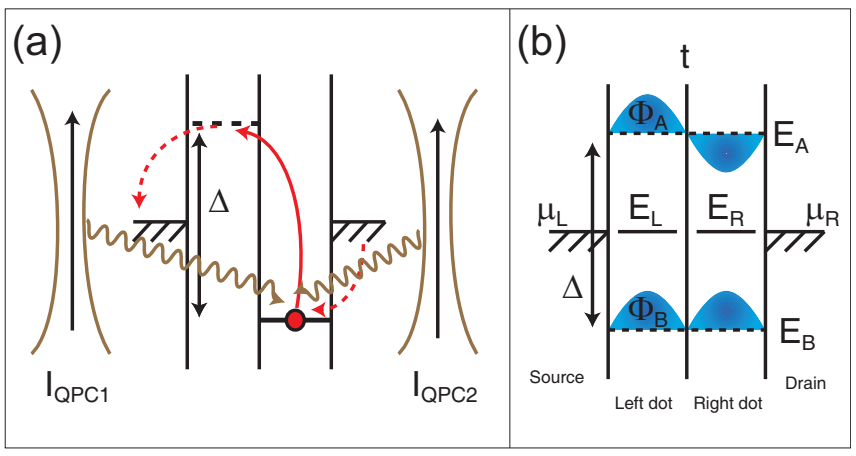

FIG. 6: (Color online) (a) Driving current through the QPC induces a current through the DQD. An electron absorbs an energy quantum $\Delta$ emitted from the left or the right QPC and is excited to the excited state. It leaves the dot via the left barrier and another electron can tunnel from the right into the right dot. (b) Schematic energy level diagram for the DQD. The wave functions of the bonding $\left(\Phi_{\mathrm{B}}\right)$ and antibonding $\left(\Phi_{\mathrm{A}}\right)$ states are shown. The electrochemical potential of the left (right) lead is $\mu_{\mathrm{L}}\left(\mu_{\mathrm{R}}\right)$.

the leads? Why do the effects of the QPCs not add up? What is the reason for the saturation of the DQD current observed in Fig. 4(a)? What is the mechanism of the energy transfer from the QPCs to the DQD? Can it explain the polarity dependence? In the next section we present a model that attempts to answer most of these questions.

\section{THE MODEL}

In the following we derive a scenario, which explains the pumping effect based on electron-phonon interaction. First, we introduce the two-level system describing the DQD. Then, we consider all possible transitions between different energy states of the DQD and express them in terms of tunneling rates. Subsequently, we derive the energy dependence of the tunneling rates. The intradot transitions are calculated in a framework of electronphonon interaction. Next, we set up a master equation and obtain the complete expression for the DQD current as a function of detuning and the temperature of the phonon bath.

Close to a pair of triple points, a double quantum dot can be regarded as a two-level system, ${ }^{2,29}$ whose bonding (ground) and antibonding (excited) states $E_{B}$ and $E_{A}$ are separated by an energy $\Delta=\sqrt{\delta^{2}+4 t^{2}}$ as shown in Fig. 6(b) ${ }^{29}$ where $t$ is tunneling coupling between the dots. The corresponding eigenvectors for bonding and antibonding states are $\left|\Psi_{\mathrm{B}}\right\rangle$ and $\left|\Psi_{\mathrm{A}}\right\rangle$. The components of the bonding and antibonding eigenstates in the basis of $\left|\phi_{\mathrm{L}}\right\rangle$ and $\left|\phi_{\mathrm{R}}\right\rangle$, the wave functions in the left $(L)$ and the right $(R)$ dot, are $c_{i, j}=\left\langle\phi_{\mathrm{i}} \mid \Psi_{\mathrm{j}}\right\rangle$, where $i=\mathrm{L}, \mathrm{R}$ and $j=\mathrm{B}, \mathrm{A}$.

As in Sec. III the detuning is defined as $\delta=E_{L}-E_{R}$ and the total energy is $E_{\text {tot }}=E_{\mathrm{L}}+E_{\mathrm{R}}$. We assume, 
that the number of the electrons in the quantum dots is fixed and its ground state energy is $E_{\mathrm{GSO}}=0$. If we add one extra electron called an excess electron the ground state energy will be $E_{\mathrm{GS} 1}=\frac{1}{2}\left(E_{\mathrm{tot}}-\Delta\right)$, while for two excess electrons, the ground state energy is $E_{\mathrm{GS} 2}=$ $\frac{1}{2}\left(E_{\mathrm{tot}}+\Delta\right)+e^{2} / C_{m}$. The corresponding electrochemical potentials are $\mu_{1}=E_{\mathrm{GS} 1}$ and $\mu_{2}=E_{\mathrm{GS} 2}-E_{\mathrm{GS} 1}$.

In the vicinity of a pair of triple points a double quantum dot can have one out of four different charge states. These different charge configurations are presented in Fig. 7 The "empty" state corresponds to a situation where there is no excess electron present in a dot and the occupation probability of this state is $p_{\mathrm{GS} 0}$. The index GS0 denotes the zero-electron ground state. In addition, one excess electron may occupy the bonding (ground) state with probability $p_{\mathrm{GS} 1}$ or the antibonding (excited) state with probability $p_{\mathrm{EX} 1}$. The last possible charge configuration is when there are two excess electrons in a double quantum dot (two-electron ground state) with occupation probability $p_{\mathrm{GS} 2}$.

The transitions between these states are determined by the tunneling rates $\Gamma_{i, j}$ and the thermal broadening of the Fermi function $f_{i, j}$ in the leads. The index $i=\mathrm{L}, \mathrm{R}$ denotes the left $(\mathrm{L})$ or the right $(\mathrm{R})$ barrier through which the electron tunnels and the index $j=0,1,2,3$ labels the transition (see Fig. 7). For example, if the dot is in a zero-electron ground state (GS0) and the electron tunnels in via the right lead, the corresponding rate is $\Gamma_{\mathrm{R}, 0} f_{\mathrm{R}, 0}$, as shown in Fig. 7. For $j=0(1)$ the Fermi function is $f_{i, 0(1)}=1 /\left\{\exp \left[\left(E_{\mathrm{B}(\mathrm{A})}-\mu_{\mathrm{i}}\right) / k_{\mathrm{B}} T_{\mathrm{F}}\right]+1\right\}$ where $k_{\mathrm{B}}$ is the Boltzmann constant and $T_{\mathrm{F}}$ is the temperature of the lead. For $j=2,3$ the expression is analogous but the energy is lifted by the mutual charging energy $e^{2} / C_{m}$.

In order to explain the experimental data presented above, we have to take into account that the tunneling rates $\Gamma_{i, j}$ do depend on the electronic wave function. They can be expressed as $\Gamma_{i, j}=\gamma_{i}\left|c_{i, j}\right|^{2}$. The coefficients $c_{i, j}$ are the left $(i=L)$ and right $(i=R)$ energy dependent components of the eigenvector of the wave function corresponding to the bonding $(j=0,3)$ and antibonding $(j=1,2)$ states. The amplitudes $\gamma_{i}$ are energyindependent parts of the $\Gamma_{i, j}$.

The rates describing the inter-dot processes, that is absorption $\Gamma_{\mathrm{abs}}$ and emission $\Gamma_{\mathrm{em}}$ are marked in Fig. 7 In the following, we assume that the double quantum dot is coupled to a bosonic bath in thermal equilibrium described by the Bose-Einstein distribution function $n_{\mathrm{B}}\left(E, T_{\mathrm{B}}\right)=1 /\left[\exp \left(E / k_{\mathrm{B}} T_{\mathrm{B}}\right)-1\right]$. The temperature $T_{\mathrm{B}}$ of this bath is determined by the current of the QPC and the base temperature of the cryostat. In case of coupling to acoustic phonons, the emission and absorption rates can be expressed as (derivation is presented in Appendix A):

$$
\Gamma_{\mathrm{em} / \mathrm{abs}}=\sum_{\lambda} \gamma_{\lambda} \Delta^{n_{\lambda}}\left(n_{\mathrm{B}}\left(\Delta, T_{\mathrm{B}}\right)+\frac{1}{2} \mp \frac{1}{2}\right) g_{\lambda}(\Delta),
$$

where the index $\lambda$ denotes piezoelectric transver-

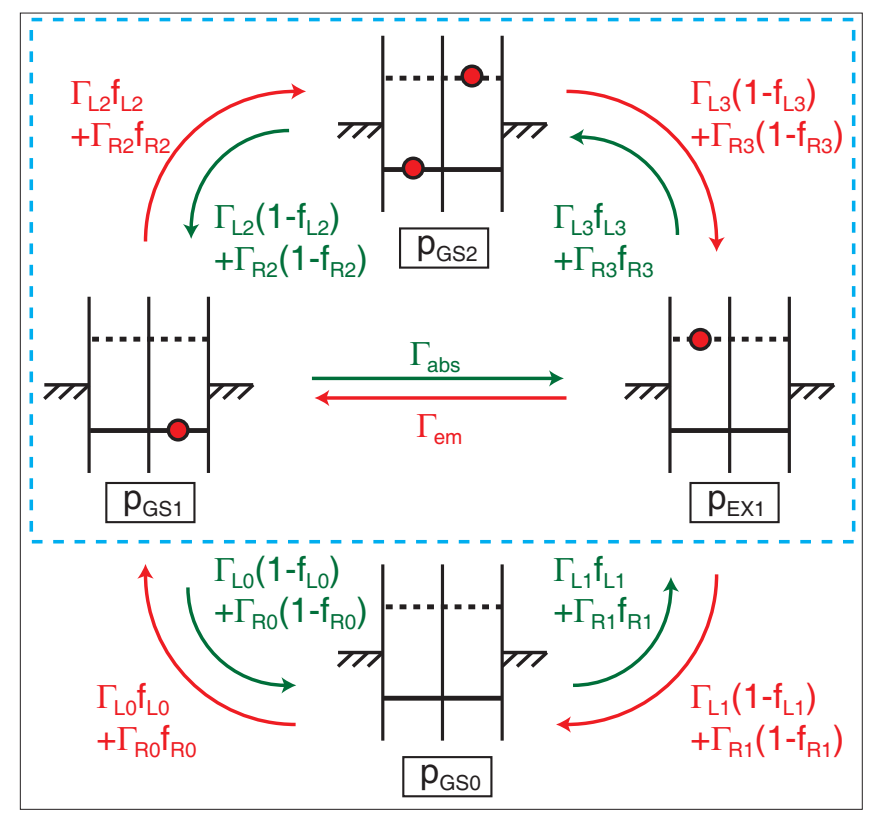

FIG. 7: (Color online) Energy level diagrams presenting four possible charge states of the DQD in the vicinity of a pair of triple points. The state GS0 corresponds to the situation with no excess electron present in the double dot. The states GS1 and EX1 are the ground and excited state of the double dot in the case of one excess electron, respectively. The fourth state GS2 is a two-excess electron ground state. The occupation probabilities of these states are labeled as $p_{\mathrm{GSO}}$, $p_{\mathrm{GS} 1}, p_{\mathrm{EX} 1}$ and $p_{\mathrm{GS} 2}$. The transitions between these states are determined by tunneling rates $\Gamma_{i, j}$ and Fermi function $f_{i, j}$. The index $i=(\mathrm{L}, \mathrm{R})$ correspond to the transition occurring through the left $i=(\mathrm{L})$ and through the right $i=(\mathrm{R})$ lead. The second index $j=0,1,2,3$ labels the transition of the electron in the DQD.

sal phonons (pe,T), piezoelectric longitudinal phonons (pe,L) or longitudinal deformation potential coupling phonons $(\mathrm{dp}, \mathrm{L})$. The exponent $n_{\lambda}$ is 1 for piezoelectric phonons and $n_{\lambda}=3$ for deformation potential

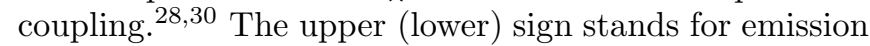
(absorption) of energy quanta. The values of the energyindependent coefficients $\gamma_{\lambda}$ are given in Appendix $\mathrm{A}$ The form-factor $g_{\lambda}(\Delta)$ is represented as:

$$
g_{\lambda}(\Delta)=c_{\mathrm{L}, \mathrm{B}}^{2} c_{\mathrm{L}, \mathrm{A}}^{2} \exp \left[-\left(\frac{\Delta r_{0}}{\hbar c_{\lambda}}\right)^{2}\right] h_{\lambda}\left(\frac{\Delta d}{\hbar c_{\lambda}}\right)
$$

where $r_{0}$ denotes the radius of a single $\mathrm{QD}, d$ is the distance between the dots, $c_{\lambda}$ is the speed of sound of $\lambda$ phonons. The complete expression for the double-dot geometry factor $h_{\lambda}\left(\Delta d / \hbar c_{\lambda}\right)$ is given in Appendix A In Eq. (2) the first factor $c_{\mathrm{L}, \mathrm{B}}^{2} c_{\mathrm{L}, \mathrm{A}}^{2}$ is related to the symmetry of the double quantum dot wave function. It suppresses transitions for the asymmetric system. The second term of Eq. (2) refers to the shape of the individual dots. It gives a high energy cut-off for phonon wavelengths much smaller than the size of a single dot $r_{0}$. The last term of Eq. (2) arises from the separation of the single dots 
in the double dot system. It suppresses small energy absorption for phonon wavelengths much larger than $d$. Only phonons with a wavelength comparable or larger than the DQD separation can interact with the electron. For large energies this term has oscillatory behavior ${ }^{20}$.

To investigate the influence of the QPCs on the dot presented in Fig. 3 in terms of rates and occupation probabilities defined in Fig. 7 we only take into account the processes surrounded by the dashed line. This is reasonable, due to a large mutual charging energy relative to the tunneling coupling. Counting the electrons passing through the right barrier leads to the following expression of the current through the double dot (the derivation is presented in Appendix (B):

$$
\begin{aligned}
I_{d o t}= & |e|\left[-p_{\mathrm{GS} 1} \Gamma_{\mathrm{R} 2} f_{\mathrm{R} 2}-p_{\mathrm{EX} 1} \Gamma_{\mathrm{R} 3} f_{\mathrm{R} 3}\right. \\
& \left.+p_{\mathrm{GS} 2} \Gamma_{\mathrm{R} 2}\left(1-f_{\mathrm{R} 2}\right)+p_{\mathrm{GS} 2} \Gamma_{\mathrm{R} 3}\left(1-f_{\mathrm{R} 3}\right)\right] .
\end{aligned}
$$

The occupation probabilities $p_{\mathrm{GS} 1}, p_{\mathrm{EX} 1}$ and $p_{\mathrm{GS} 2}$ are functions of $\Gamma_{i, j}$ and $f_{i, j}$ resulting from a stationary solution of the master equation (see Appendix $(\mathrm{B})$ ).

\section{RESULTS AND INTERPRETATION}

We used expression (3) to fit the data shown in Fig. 3 During the fitting procedure, the 8 traces shown in Fig. 3(a) and (b) and an additional set of 16 traces being a combination of $I_{\mathrm{QPC} 1,2}=0, \pm 50, \pm 75, \pm 100 \mathrm{nA}$ were fitted simultaneously. The following fitting parameters were shared: amplitudes $\gamma_{\mathrm{L}}, \gamma_{\mathrm{R}}$ and tunneling coupling $t$. The only parameter specific for each trace was the temperature of the phonon bath $T_{\mathrm{b}}$.

The fit represents the overall shape of the measured data very well. The extracted tunneling coupling is $t=50 \mu \mathrm{eV}$. In Fig. 2(a) the grey (green online) solid line is the calculated boundary between the honeycomb cells assuming a tunneling coupling of $50 \mu \mathrm{eV}$, the black (red online) crosses are the maxima position of the DQD current peaks and the black dashed line is the boundary assuming $t=0$. Unfortunately, the sample was not stable enough to map a stability diagram with a resolution high enough to determine the tunneling coupling directly. However, the $t$ obtained from the fits seems to be reasonable and matches with the data presented on the stability diagram.

The temperature of the phonon bath $T_{\mathrm{B}}$ obtained from the fits varies from $0.6 \mathrm{~K}$ (blue trace in Fig. 3(a)) to $1.2 \mathrm{~K}$ (red trace in Fig. 3(b)). The difference between the temperatures $T_{\mathrm{b}}$ and the electronic temperature $T_{\mathrm{f}}=100 \mathrm{mK}$ gives rise to the DQD current.

The extracted amplitude $\gamma_{\mathrm{R}}$ is of the order of $7.8 \mathrm{MHz}$ and $\gamma_{\mathrm{L}}$ is about $0.5 \mathrm{GHz}$. This is in agreement with our previous statement that the right barrier is more opaque than the left one. This leads to a deviation from perfect antisymmetry of the dot current along the detuning line, i.e., to suppressed dot current for negative detuning.

The tail of the curve at large detuning is mainly determined by the amplitudes $c_{\mathrm{L}, \mathrm{B}}^{2} c_{\mathrm{L}, \mathrm{A}}^{2}$ present in Eq. (2) that drop to zero like $t^{2} / \delta^{2}$ and the Bose-Einstein distribution function given in Eq. (11). Although, the real distribution of the bosonic environment is not necessarily equilibrated and could have another form, the qualitative agreement with the data does not depend strongly on the details of this distribution.

Using the parameters obtained in the fits, we calculated the current as a function of the gate voltages using the model containing all four charge states presented in Fig. 7 In the absence of the QPC current $\left(T_{\mathrm{b}}=T_{f}=70 \mathrm{mK}\right)$, the result is shown in Fig. 2 (c). The asymmetry and magnitude of the current along the upper dashed line is reproduced very well. This is not the case for the lower branch indicating transitions $(\mathrm{M}, \mathrm{N}) \leftrightarrow(\mathrm{M}+1, \mathrm{~N})$. This may be due to a change of $\gamma_{\mathrm{L}}$ and $\gamma_{R}$, which we assumed to be constant in our model but which may change in the experiment. For $1 \mathrm{mV}$ DC bias across the QPC2 (Fig. 2(b)), the bosonic temperature is around $T_{\mathrm{b}}=0.8 \mathrm{~K}$. The corresponding calculations are shown in Fig. 2(d). The red triangle indicates the region with the QPC induced current that is in agreement with the measured data in (b).

To investigate the dependence of the phonon temperature on the QPC current we calculated $T_{\mathrm{b}}$ for every point from Fig. 4(a) using the values obtained in previous fits. The results are shown in Fig. 4(b). For small QPC currents the error bars are large and no clear dependence is visible. For QPC currents above $50 \mathrm{nA}$ the dependence is quadratic $T_{\mathrm{b}} \sim I_{\mathrm{QPC}}^{2}$, which means that the temperature of the bosonic bath is proportional to the power emitted by the QPC. The reconstructed temperatures corresponding to the measurement presented in Fig. 5(a) are plotted in Fig. 5(b). For very small DQD current it is impossible to estimate the temperature of the phonon bath with sufficient accuracy.

The saturation of the DQD current for large QPC currents cannot be attributed to the high occupation probability of the antibonding state $p_{\mathrm{EX} 1}$. We have estimated that the $p_{\mathrm{EX} 1}$ value does not exceed a few percent and most of the time the dot is occupied by one electron in its ground state. The maximum current is determined by the right tunneling barrier.

A series of experiments reporting the observation of a DQD current induced by a single and independently biased QPC, is described in Refs. 15, 16, 17. These experiments were performed in the regime of large DQD current, strong bias voltage applied to the QPC and large tunneling coupling, which is similar to our situation here. The DQD current was related to inelastic relaxation of electrons in partly transmitting 1D channels of the $\mathrm{QPC}=15$ and qualitatively consistent with an energy transfer mechanism based on nonequilibrium acoustic phonons. ${ }^{16,17}$ In contrast to our experimental data, in these experiments the DQD current was large when the conductance of the QPC was tuned to $e^{2} / h$ and strongly suppressed in plateau regions.

Recent research has proven that the absorption of a photon can be the dominant process $\frac{18,27}{}$ in similar sit- 
uations. However, these time-resolved experiments were performed in a different regime, where the dominant tunneling rates are of order of $1 \mathrm{kHz}$, whereas in our system the double quantum dot is much more strongly coupled to the leads. Another difference is the presence of a $\mathrm{Ti}$ top-gate in our structure, that screens the electrostatic interaction between the DQD and the QPCs. Due to the lower sensitivity of direct dot current measurements compared to the time-resolved technique it is not possible to observe the gap in the DQD current when $\left|e V_{Q P C}\right|<\delta$. Calculations of the emission and absorption rates in a DQD induced by electron-photon interaction show, that the effect is irrelevant compared to the emission and absorption of phonons discussed here. 30

We have also tested the possibility that the entire dot current in the region forbidden by the Coulomb blockade is due to a thermopower effect induced by different temperatures in the source and the drain. We found, that it would be only possible if the temperature difference between source and drain lead was larger than $1 \mathrm{~K}$ for a QPC current of $100 \mathrm{nA}$, which is one order of magnitude larger than expected. ${ }^{31}$ Another argument against a thermopower model is that always the drain lead would have to be warmer, even if the far QPC1 (that couples better to the drain lead) was biased. Even so, the thermopower model did not describe the data as well as the emission/absorption model.

\section{CONCLUSIONS}

We have presented the influence of two independent quantum point contacts on a double quantum dot. A number of of questions arising during the investigation and presented in Sec. [V] could be answered.

In the first place, we established the possible dominant mechanism of energy transfer between the double quantum dot and the quantum point contact in the investigated regime. Driving current through the QPC leads to emission of energy that increases the temperature of the bosonic environment. We identify these bosons as acoustic phonons. To model the interaction of the phonons with the double quantum dot we have assumed that their energy distribution is described by Bose-Einstein statistics.

Another important point is a non-additive effect of both QPC currents. It is understood in terms of the temperature of the bosonic bath. We find that the DQD current is proportional to the power emitted by the QPCs.

Next, we interpreted the leveling off of the DQD current as a QPC current is increased. For large QPC powers (above $0.1 \mathrm{nW}$ ) the temperature of the phonon bath increases linearly. The observed saturation of the current is due to the finite transparency of the tunneling barriers and not to the high occupation probabilities $p_{E X 1}$.

The polarity dependence (Fig. 4) cannot be explained within the discussed model and its origin remains to be investigated. It would be interesting to further investi- gate this effect, for example, by using different geometrical arrangements.

Finally, we observed strong deviation from perfect antisymmetry of the dot current along the detuning line when the QPC current is driven. It can be attributed to the asymmetry of the source and drain barriers.

All the measurements were performed with both QPCs tuned to their first plateau. Thus we can exclude the influence of shot-noise phenomena in the quantum point contacts.

\section{APPENDIX A: ABSORPTION AND EMISSION RATES IN A DQD INDUCED BY ELECTRON-PHONON INTERACTION}

Generally, the emission and absorption rates can be expressed using Fermi's golden rule:

$$
\Gamma_{\mathrm{em} / \mathrm{abs}}=\frac{2 \pi}{\hbar} \sum_{\mathbf{q}, \lambda}\left|\left\langle\Phi_{\mathrm{B}}\left|H_{\mathrm{e}-\mathrm{p}}(\mathbf{r})\right| \Phi_{\mathrm{A}}\right\rangle\right|^{2} \delta\left(\Delta-\hbar \omega_{\mathbf{q}, \lambda}\right),
$$

where the sum extends over all wave vectors $\mathbf{q}$. The index $\lambda$ denotes the type of acoustic phonons and their coupling in GaAs: piezoelectric longitudinal (pe,L), piezoelectric transversal (pe,T) and longitudinal, deformation potential coupling $(\mathrm{dp}, \mathrm{L})$. The phonons have linear dispersion relation $\omega_{\mathbf{q}, \lambda}=c_{\lambda}|\mathbf{q}|$. $\Phi_{\mathrm{B}}$ and $\Phi_{\mathrm{A}}$ are wave functions of bonding and antibonding states separated by energy $\Delta$. The interaction hamiltonian $H_{\mathrm{e}-\mathrm{p}}$ can be written as a sum of piezoelectric interaction $H_{\mathrm{pe}}$ and deformation potential coupling $H_{\mathrm{dp}}$ :

$$
H_{\mathrm{e}-\mathrm{p}}(\mathbf{r})=H_{\mathrm{pe}}(\mathbf{r})+H_{\mathrm{dp}}(\mathbf{r}),
$$

with

$$
\begin{aligned}
H_{\mathrm{pe}}(\mathbf{r}) & =-\frac{|e| d_{14}}{2 \epsilon \epsilon_{0} \sqrt{N M}} \sum_{\mathbf{q}, \lambda}\left(\frac{\hbar}{2 \omega_{\mathbf{q}, \lambda}}\right)^{1 / 2} F(\mathbf{q}) e^{i \mathbf{q r}} \\
& \times\left(a_{\mathbf{q}, \lambda}+a_{-\mathbf{q}, \lambda}^{\dagger}\right) \\
H_{\mathrm{dp}}(\mathbf{r}) & =-\frac{-i D}{\sqrt{N M}} \sum_{\mathbf{q}}\left(\frac{\hbar}{2 \omega_{\mathbf{q}, \lambda}}\right)^{1 / 2}|\mathbf{q}| e^{i \mathbf{q r}} \\
& \times\left(a_{\mathbf{q}, \lambda}+a_{-\mathbf{q}, \lambda}^{\dagger}\right)
\end{aligned}
$$

In above equations $d_{14}$ is an element of piezoelectric tensor, $\epsilon_{0}$ is the vacuum permittivity, $\epsilon$ is the dielectric constant, $N$ is the number of atoms in the crystal, $\mathrm{M}$ is the atomic mass and D denotes deformation potential coupling constant. The dimensionless function $F(\mathbf{q})$ has form:

$$
F(\mathbf{q})=\frac{1}{|\mathbf{q}|^{2}} \sum_{i k l}\left|\varepsilon_{i k l}\right|\left(e_{\lambda, k} q_{l}+e_{\lambda, l} q_{k}\right) q_{i}
$$

where $\varepsilon_{i k l}$ is the Levi-Civita symbol and $e_{\lambda, i}$ is the $i$ component of the eigenvector associated with mode $\lambda$. 
Inserting Eq. (A2) into Eq. (A1) leads to the following expression:

$$
\begin{aligned}
\Gamma_{\mathrm{em} / \mathrm{abs}} & =\left(\gamma_{\mathrm{pe}} \Delta\left[g_{\mathrm{pe}, \mathrm{T}}(\Delta)+g_{\mathrm{pe}, \mathrm{L}}(\Delta)\right]+\gamma_{\mathrm{dp}} \Delta^{3} g_{\mathrm{dp}}(\Delta)\right) \\
& \times\left(n(\Delta)+\frac{1}{2} \mp \frac{1}{2}\right)
\end{aligned}
$$

where the upper sign refers to phonon absorption and the lower to phonon emission. The constants $\gamma_{\mathrm{pe}}$ and $\gamma_{\mathrm{dp}}$ are given by:

$$
\begin{aligned}
& \gamma_{\mathrm{pe}}=\frac{|e|^{2} d_{14}^{2}}{2 \pi\left(2 \epsilon \epsilon_{0}\right)^{2} \rho \hbar^{2} c_{\lambda}^{3}}=5 \times 10^{10} \mathrm{meV}^{-1} \mathrm{~s}^{-1}, \\
& \gamma_{\mathrm{dp}}=\frac{D^{2}}{2 \pi \hbar^{4} c_{\lambda}^{5} \rho}=7.3 \times 10^{11} \mathrm{meV}^{-3} \mathrm{~s}^{-1}
\end{aligned}
$$

where $\rho$ is a density of the GaAs crystal. The energy dependent functions $g_{\mathrm{pe}}(\Delta)$ and $g_{\mathrm{dp}}(\Delta)$ are defined as:

$$
\begin{aligned}
g_{\mathrm{pe}}(\Delta) & =\sum_{\lambda} \frac{\hbar^{3} c_{\lambda}^{3}}{4 \pi \Delta^{2}} \int d^{3} q F_{\lambda}^{2}( \pm \mathbf{q}) \\
& \times\left|\left\langle\Phi_{\mathrm{B}}\left|e^{ \pm i \mathbf{q r}}\right| \Phi_{\mathrm{A}}\right\rangle\right|^{2} \delta\left(\Delta-\hbar \omega_{\mathbf{q}, \lambda}\right), \\
g_{\mathrm{dp}}(\Delta) & =\frac{\hbar^{5} c_{\lambda}^{5}}{4 \pi \Delta^{4}} \int d^{3} q|\mathbf{q}|^{2} \\
& \times\left|\left\langle\Phi_{\mathrm{B}}\left|e^{ \pm i \mathbf{q r}}\right| \Phi_{\mathrm{A}}\right\rangle\right|^{2} \delta\left(\Delta-\hbar \omega_{\mathbf{q}, \lambda}\right) .
\end{aligned}
$$

Assuming negligible overlap between the wave functions of the two dots and taking a gaussian-shaped singleelectron wave function, the matrix element is found to be:

$$
\left|\left\langle\Phi_{\mathrm{B}}\left|e^{ \pm i \mathbf{q r} \mathbf{r}}\right| \Phi_{\mathrm{A}}\right\rangle\right|^{2}=2 c_{\mathrm{L}, \mathrm{B}}^{2} c_{\mathrm{L}, \mathrm{A}}^{2} e^{-\left(\mathbf{q r}_{\mathbf{o}}\right)^{2}}[1-\cos (\mathbf{q d})],
$$

where $d$ is the distance between the dots and $r_{0}$ is the radius of a single dot. Inserting (A6) into Eqs. (A5) gives:

$$
g_{\lambda}(\Delta)=c_{\mathrm{L}, \mathrm{B}}^{2} c_{\mathrm{L}, \mathrm{A}}^{2} e^{-\left(\mathbf{q r}_{\mathbf{0}}\right)^{2}} h_{\lambda}\left(\frac{\Delta d}{\hbar c_{\lambda}}\right)
$$

For piezoelectric transversal phonons the above expression was calculated by averaging the function $f_{\mathrm{pe}, \mathrm{T}}(\mathbf{q})$ over all possible transversal directions. The geometry factors $h_{\lambda}\left(\eta=\Delta d / \hbar c_{\lambda}\right)$ are given by:

$$
\begin{aligned}
h_{\mathrm{pe}, \mathrm{L}}(\eta) & =\frac{24}{35}+72 \eta^{-7}\left[9 \eta\left(\eta^{2}-10\right) \cos \eta\right. \\
& \left.+\left(\eta^{4}+39 \eta^{2}+90\right) \sin \eta\right], \\
h_{\mathrm{pe}, \mathrm{T}}(\eta) & =\frac{32}{35}+16 \eta^{-7}\left[\eta\left(\eta^{4}-51 \eta^{2}+405\right) \cos \eta\right. \\
& \left.-3\left(3 \eta^{4}-62 \eta^{2}+135\right) \sin \eta\right], \\
h_{\mathrm{dp}, \mathrm{L}}(\eta) & =2-2 \eta^{-1} \sin \eta .
\end{aligned}
$$

Combining Eq. (A8) with Eq. (A7) and inserting the result into Eq. (A44) gives a complete expression for the absorption and emission rates.

\section{APPENDIX B: RATE EQUATION}

To relate the DQD current to the tunneling rates we write down the rate equation for the occupation of the states:

$$
\frac{d}{d t}\left[\begin{array}{l}
p_{\mathrm{GS} 1} \\
p_{\mathrm{EX} 1} \\
p_{\mathrm{GS} 2}
\end{array}\right]=\left[\begin{array}{ccc}
-\left(\Gamma_{\mathrm{abs}}+A_{2}\right) & \Gamma_{\mathrm{em}} & B_{2} \\
\Gamma_{\mathrm{abs}} & -\left(\Gamma_{\mathrm{em}}+A_{3}\right) & B_{3} \\
A_{2} & A_{3} & -\left(B_{2}+B_{3}\right)
\end{array}\right]\left[\begin{array}{l}
p_{\mathrm{GS} 1} \\
p_{\mathrm{EX} 1} \\
p_{\mathrm{GS} 2}
\end{array}\right],
$$

with additional condition $p_{\mathrm{GS} 1}+p_{\mathrm{EX} 1}+p_{\mathrm{GS} 2}=1$. The terms $A_{j}$ and $B_{j}$ are defined as:

$$
\begin{aligned}
A_{j} & =\sum_{i=\mathrm{L}, \mathrm{R}} \Gamma_{i, j} f_{i, j} \\
B_{j} & =\sum_{i=\mathrm{L}, \mathrm{R}} \Gamma_{i, j}\left(1-f_{i, j}\right) .
\end{aligned}
$$

To find the expression for the current flowing through a $\mathrm{DQD}$, we take the right barrier as a current reference. It means that, if an electron passes the right barrier to the left (right), its contribution to the DQD current is positive (negative).

$$
\begin{aligned}
I_{d o t}= & |e|\left[-p_{\mathrm{GS} 1} \Gamma_{\mathrm{R} 2} f_{\mathrm{R} 2}-p_{\mathrm{EX} 1} \Gamma_{\mathrm{R} 3} f_{\mathrm{R} 3}\right. \\
& \left.+p_{\mathrm{GS} 2} \Gamma_{\mathrm{R} 2}\left(1-f_{\mathrm{R} 2}\right)+p_{\mathrm{GS} 2} \Gamma_{\mathrm{R} 3}\left(1-f_{\mathrm{R} 3}\right)\right],
\end{aligned}
$$

The first (second) term of Eq. (B3) corresponds to the electrons moving from the bonding (antibonding) state to the right lead and the third and fourth term to the electrons entering the ground or excited state of the dot from the right lead. By inserting a stationary solution of Eq. (B1) into Eq. (B3) one obtains an expression for the steady state DQD current. 
1 M. Reed, J. Randall, J. Luscombe, W. Frensley, R. Aggarwal, R. Matyi, T. Moore, and A. Wetsel, in Festkörperprobleme 29 (Springer Berlin/Heidelberg, 1989), pp. 267-283.

2 W. G. van der Wiel, S. De Franceschi, J. M. Elzerman, T. Fujisawa, S. Tarucha, and L. P. Kouwenhoven, Rev. Mod. Phys. 75, 1 (2002).

${ }^{3}$ R. Hanson, L. P. Kouwenhoven, J. R. Petta, S. Tarucha, and L. M. K. Vandersypen, Rev. Mod. Phys. 79, 1217 (2007).

4 T. Fujisawa and S. Tarucha, Jpn. J. of App. Phys. 36, 4000 (1997).

5 T. Fujisawa and S. Tarucha, Superlattices and Microstructures 21, 247 (1997).

6 T. H. Oosterkamp, L. P. Kouwenhoven, A. E. A. Koolen, N. C. van der Vaart, and C. J. P. M. Harmans, Phys. Rev. Lett. 78, 1536 (1997).

7 T. H. Oosterkamp, T. Fujisawa, W. G. van der Wiel, K. Ishibashi, R. V. Hijman, S. Tarucha, and L. P. Kouwenhoven, Nature 395, 873 (1998).

8 W. G. van der Wiel, T. Fujisawa, T. H. Oosterkamp, and L. P. Kouwenhoven, Physica B 272, 31 (1999).

${ }^{9}$ H. Qin, A. W. Holleitner, K. Eberl, and R. H. Blick, Phys. Rev. B 64, 241302 (R) (2001).

10 M. Switkes, C. M. Marcus, K. Campman, and A. C. Gossard, Science 283, 1905 (1999).

11 G. Platero and R. Aguado, Physics Reports 395, 1 (2004).

12 M. Field, C. G. Smith, M. Pepper, D. A. Ritchie, J. E. F. Frost, G. A. C. Jones, and D. G. Hasko, Phys. Rev. Lett. 70, 1311 (1993).

13 J. M. Elzerman, R. Hanson, J. S. Greidanus, L. H. Willems van Beveren, S. De Franceschi, L. M. K. Vandersypen, S. Tarucha, and L. P. Kouwenhoven, Phys. Rev. B 67, 161308 (R) (2003).

14 L. DiCarlo, H. J. Lynch, A. C. Johnson, L. I. Childress, K. Crockett, C. M. Marcus, M. P. Hanson, and A. C. Gossard, Phys. Rev. Lett. 92, 226801 (2004).

15 V. S. Khrapai, S. Ludwig, J. P. Kotthaus, H. P. Tranitz, and W. Wegscheider, Phys. Rev. Lett. 97, 176803 (2006).

16 V. S. Khrapai, S. Ludwig, J. P. Kotthaus, H. P. Tranitz, and W. Wegscheider, Physica E: Low-dimensional Systems and Nanostructures 40, 995 (2008).
17 V. S. Khrapai, S. Ludwig, J. P. Kotthaus, H. P. Tranitz, and W. Wegscheider (2008), arXiv:0805.0724v1.

18 S. Gustavsson, M. Studer, R. Leturcq, T. Ihn, K. Ensslin, D. C. Driscoll, and A. C. Gossard, Phys. Rev. Lett. 99, 206804 (2007).

19 T. Fujisawa, T. Hayashi, S. Jung, Y.-H. Jeong, and Y. Hirayama, in Quantum Computing in Solid State Systems (Springer, 2006), pp. 279-287.

20 T. Fujisawa, T. H. Oosterkamp, W. G. van der Wiel, B. W. Broer, R. Aguado, S. Tarucha, and L. P. Kouwenhoven, Science 282, 932 (1998).

21 E. Onac, F. Balestro, L. H. Willems van Beveren, U. Hartmann, Y. V. Nazarov, and L. P. Kouwenhoven, Phys. Rev. Lett. 96, 176601 (2006).

22 E. Zakka-Bajjani, J. Segala, F. Portier, P. Roche, D. C. Glattli, A. Cavanna, and Y. Jin, Phys. Rev. Lett. 99, 236803 (2007).

23 M. Sigrist, A. Fuhrer, T. Ihn, K. Ensslin, D. C. Driscoll, and A. C. Gossard, Appl. Phys. Lett. 85, 3558 (2004).

24 A. Fuhrer, A. Dorn, S. Luscher, T. Heinzel, K. Ensslin, W. Wegscheider, and M. Bichler, Superlattices and Microstructures 31, 19 (2002).

25 J. H. Davies, I. A. Larkin, and E. V. Sukhorukov, J. of App. Phys. 77, 4504 (1995).

26 In order to estimate the electrostatic potential in a $2 \mathrm{DEG}$ plane created by the AFM-defined oxide lines and titanium top-gates, we treated the oxide lines as patterned metalic gates covering the surface of the semiconductor heterostructure. The Ti top-gates were definied in the same manner. In our calculations the voltage applied to the gates corresponding to the oxide lines was 10 times larger than the voltage applied to the Ti top-gates.

27 S. Gustavsson, I. Shorubalko, R. Leturcq, S. Schön, and K. Ensslin, App. Phys. Lett. 92, 152101 (2008).

28 U. Bockelmann, Phys. Rev. B 50, 17271 (1994).

29 R. H. Blick, D. Pfannkuche, R. J. Haug, K. v. Klitzing, and K. Eberl, Phys. Rev. Lett. 80, 4032 (1998).

30 T. Ihn, unpublished.

31 S. Gustavsson, Ph.D. thesis, ETH Zurich, Zurich, Switzerland (2008). 